\title{
Dinámica del manglar en el complejo lagunar de Cispatá (Caribe colombiano) en los últimos 900 años
}

\author{
Ana Castaño ${ }^{1}$ Ligia Urrego $^{1} \&$ Gladys Bernal $^{2}$ \\ 1. Departamento de Ciencias Forestales, Universidad Nacional de Colombia, Medellín, Colombia, Calle 59 A No. 63-20, \\ Autopista Norte; leurrego@unal.edu.co,arcastan@unal.edu.co \\ 2. Escuela de Geociencias y Medio Ambiente, Universidad Nacional de Colombia, Medellín, Colombia, Cra 80 No \\ 65-223, M2-319; gbernal@unal.edu.co
}

Recibido 23-X-2009. Corregido 18-V-2010. Aceptado 17-VI-2010.

\begin{abstract}
Mangrove dynamics in the Cispata lagoon system (Colombian Caribbean) during last 900 years. The lagoon complex of Cispatá (old Sinú river delta) located at the Northwestern coast of the Colombian Caribbean, encloses one of the biggest mangrove areas in this region. This area has changed during the last 330 years because of several environmental and climatic causes, mainly changes in the position of the delta (Sinú River), which is the main freshwater source in this area, and sea level rise. We hypothesized that the climatic and geomorphologic dynamics has caused changes in the extension and composition of mangrove vegetation, especially during last 150 years. The dynamics of mangroves during the last 900 years was reconstructed based on the changes in the stratigraphy, pollen record, calcite concentrations $\left(\mathrm{CaCO}_{3}\right)$ and $\mathrm{C} / \mathrm{N}$ ratio, along two sediment cores from La Flotante and Navio lagoons, located in Cispatá complex. The age model was built based on lineal interpolation of ${ }^{210} \mathrm{~Pb}$ ages and changes in granulometry. Establishment and expansion of mangrove forests during the last 900 years were related to fluviomarine dynamics in the area and the lagoon formation. During the period encompassed between 1064 and 1762 A.D., the Mestizos spit was formed when marine conditions predominated in the surroundings of La Flotante Lagoon. At the site of Navío, a river dominated lagoon, terrigenous conditions dominated since 1830. Although the colonization of herbaceous pioneer vegetation started between 1142 and 1331 A.D., mangrove colonization only took place since 1717 A.D. Mangrove colonization was a result of the delta progradation. In 1849 A.D. the Sinú river delta migrated to the Cispatá bay. The eustatic sea level rise, the increase in river discharges and sedimentation rates produced the establishment of mangrove forests dominated by Rhizophora since 1849. Since 1900 a marine intrusion was recorded in both lagoons. In 1938 , the migration of the delta toward its actual location in Tinajones gave place to the formation of the present lagoon system and to the expansion of mangrove forests, which reflects the balance between the high alluvial sediment input and the current sea level rise as has been recorded in similar ecosystems. Rev. Biol. Trop. 58 (4): 1347-1366. Epub 2010 December 01.
\end{abstract}

Key words: mangroves, palinology, coastal lagoon, Cispatá, Colombian Caribbean.

Los manglares ocupan la cuarta parte de las costas tropicales del mundo y se consideran entre las reservas ecológicas y forestales del planeta (Field 1995). Su alta productividad, así como su estructura y distribución, están determinadas principalmente por el aporte de agua dulce y sedimentos transportados por los ríos, la salinidad, la geomorfología, la estacionalidad de las lluvias y la amplitud y duración de las mareas, entre otros (Field 1995, Sánchez-
Páez et al. 1997, Hogarth 1999, Ellison et al. 2000, Willard et al. 2001). Los manglares protegen las costas del oleaje, controlan la erosión y son fuente de múltiples productos para las comunidades humanas de las costas (Ellison 1996, Mazda et al. 2002, Gilman et al. 2007, Alongi 2008).

Las variaciones ambientales asociadas a los cambios climáticos afectan el establecimiento y supervivencia de los manglares y 
pueden causar aumento o disminución del área cubierta por estos ecosistemas. Los cambios en las temperaturas superficiales, las oscilaciones en el nivel del mar, los procesos erosivos, el incremento en la frecuencia y magnitud de las perturbaciones ciclónicas y la dinámica fluvial, han generado cambios en la distribución de los manglares del Caribe (Field 1995, Snedaker 1995, Gilman et al. 2007, Ellison 2008). Actualmente, los manglares se consideran entre los ecosistemas más vulnerables a los efectos del cambio climático global (IPCC 2007). Pero justamente la sensibilidad a los cambios ambientales hace de los manglares buenos indicadores ambientales (Rull 1998, Kumaran et al. 2007). Adicionalmente, su composición florística relativamente simple y la adaptación de las especies de mangle a diferentes niveles de salinidad, permiten que estos ecosistemas sean fácilmente identificables en los registros palinológicos, constituyéndose en indicadores potenciales de cambios ambientales tanto a nivel regional (cambios en el nivel del mar), y locales (geomorfológicos, en la salinidad, en el drenaje, y en el grado de inundación) (Blasco et al. 1996, Gilman et al. 2007).

Las reconstrucciones paleoecológicas en el Caribe y en gran parte del cinturón tropical y subtropical han permitido establecer los cambios de los manglares y su concordancia con los cambios ambientales, especialmente los cambios en el nivel relativo del mar a diferentes escalas temporales, por medio del análisis de polen en columnas de sedimentos (Rull 1998, 1999, Nyberg et al. 2001, Berger et al. 2006, Carvalho et al. 2006, Engelhart et al. 2007, Ferreira et al. 2007, Ellison 2008). Las investigaciones en manglares han establecido que desde el Holoceno medio al tardío los niveles del mar se incrementaron gradualmente y los manglares permanecieron en muchas áreas, en donde se logró mantener la elevación del sustrato por el aporte de sedimentos desde las áreas continentales y de detritos orgánicos para contrarrestar este efecto (Parkinson et al.1994, Ellison 2008). Sin embargo en otras áreas, los manglares disminuyeron debido al rápido incremento del nivel del mar, la erosión costera derivada de éste y las bajas tasas de sedimentación (Ellison 2008).

En América los manglares actuales se establecieron después de 3000 A.P. cuando ocurrió una estabilización en el ascenso del nivel del mar después del óptimo holocénico (Ellison 1996); sin embargo, cambios climáticos posteriores, especialmente durante el último milenio, también generaron cambios importantes en su distribución. Entre estos se han identificado reducciones en las coberturas de manglar relacionadas con regresiones marinas durante la Pequeña Edad de Hielo -PEH (1550-1850 A.D.) en las cuales se registraron menores precipitaciones y menores caudales de los ríos, lo que a su vez implicó menor aporte de sedimentos (Nyberg et al. 2002, Cohen et al. 2005). Igualmente, se han registrado migraciones tierra adentro de los manglares durante las últimas décadas, asociadas al actual incremento en el nivel del mar, iniciado al culminar la PEH (Cohen et al. 2005).

Investigaciones paleoecológicas realizadas en el Caribe colombiano, han mostrado la respuesta de los manglares a fenómenos locales de subsidencia, variaciones en la dinámica fluvial, así como a cambios en el nivel del mar y la actividad antrópica durante el Holoceno tardío (Castaño 2004, González et al. 2010). Sin embargo, aún no se conoce la significancia regional de estos fenómenos y quedan muchas preguntas acerca de la respuesta de los manglares a los cambios ambientales recientes; en especial, no se sabe en qué proporción la evolución de los manglares responde a los cambios climáticos, los geomorfológicos o al efecto combinado de ambos.

Los manglares del complejo lagunar Cispatá cubren aproximadamente $150 \mathrm{~km}^{2}$ y constituyen la segunda área en extensión cubierta por estos ecosistemas en el Caribe colombiano (Robertson \& Chaparro 1998, Serrano 2004). El área ha presentado una gran dinámica por efecto de los cambios en el delta del río Sinú, fuente principal de agua dulce de este complejo, el cual ha migrado varias veces su posición en los últimos 330 años. En esta investigación postulamos que la variabilidad climática y 
la dinámica geomorfológica, han influido en los procesos de colonización, distribución y composición de los manglares de Cispatá en el último milenio, especialmente en los últimos 150 años.

\section{MATERIALES Y MÉTODOS}

Area de estudio: Las secciones de sedimentos se obtuvieron de las lagunas La Flotante $\left(9^{\circ} 23^{\prime} 37^{\prime \prime} \mathrm{N}, 75^{\circ} 48^{\prime} 49^{\prime \prime} \mathrm{O}\right)$ y Navío (9'24'19" N, 75'51'41' O); localizadas en la zona baja de la cuenca del río Sinú, en El Complejo Lagunar Cispatá, Noroeste de Colombia (Fig. 1). El río Sinú nace en el extremo Norte de la Cordillera Occidental de los Andes, en el Nudo de Paramillo, a más de 3 000m.s.n.m. (Fig. 1b) y tiene una extensión aproximada de 380km (Hernández \& Wilches 1990, Robertson \& Chaparro 1998, Atencio-García 2000, Díaz et al. 2000).

El delta del río Sinú ha cambiado su posición seis veces desde $330 \pm 30$ años A.C. El último cambio lo registró en 1938 A.D. cuando se ubicó en su posición actual (Tinajones). En las tres últimas ubicaciones del delta denominadas Venados, Mestizos y Cispatá, éste permaneció estable más o menos 100 años en cada una de ellas (Robertson \& Chaparro 1998, Serrano 2001).

Cerca de un tercio de la cuenca del río Sinú, es decir unos $4583 \mathrm{~km}^{2}$ está constituida por pendientes empinadas, el resto de la cuenca está formada por tierras bajas, planas a onduladas o cenagosas, que ayudan a regular el caudal del río (Parsons 1952, Palacio \& Restrepo 1999). Cerca del delta de Tinajones el caudal medio del río es de $383 \mathrm{~m}^{3} / \mathrm{s}$, con máximos de $715 \mathrm{~m}^{3} / \mathrm{s}$ y mínimos de $240 \mathrm{~m}^{3} / \mathrm{s}$ (Molina et al. 1994, Jurado \& Liebmann 2003, Serrano 2004). Estos caudales han registrado variaciones interanuales relacionadas con eventos ENSO, con anomalías positivas durante años Niña y negativas durante años Niño (RuízOchoa et al. 2008).

La parte baja de la cuenca presenta un régimen de precipitación unimodal, con un promedio anual de $1338 \mathrm{~mm} / \mathrm{año}$; en la época seca (diciembre-abril) las precipitaciones no superan $50 \mathrm{~mm} / \mathrm{mes}$, mientras que entre agosto

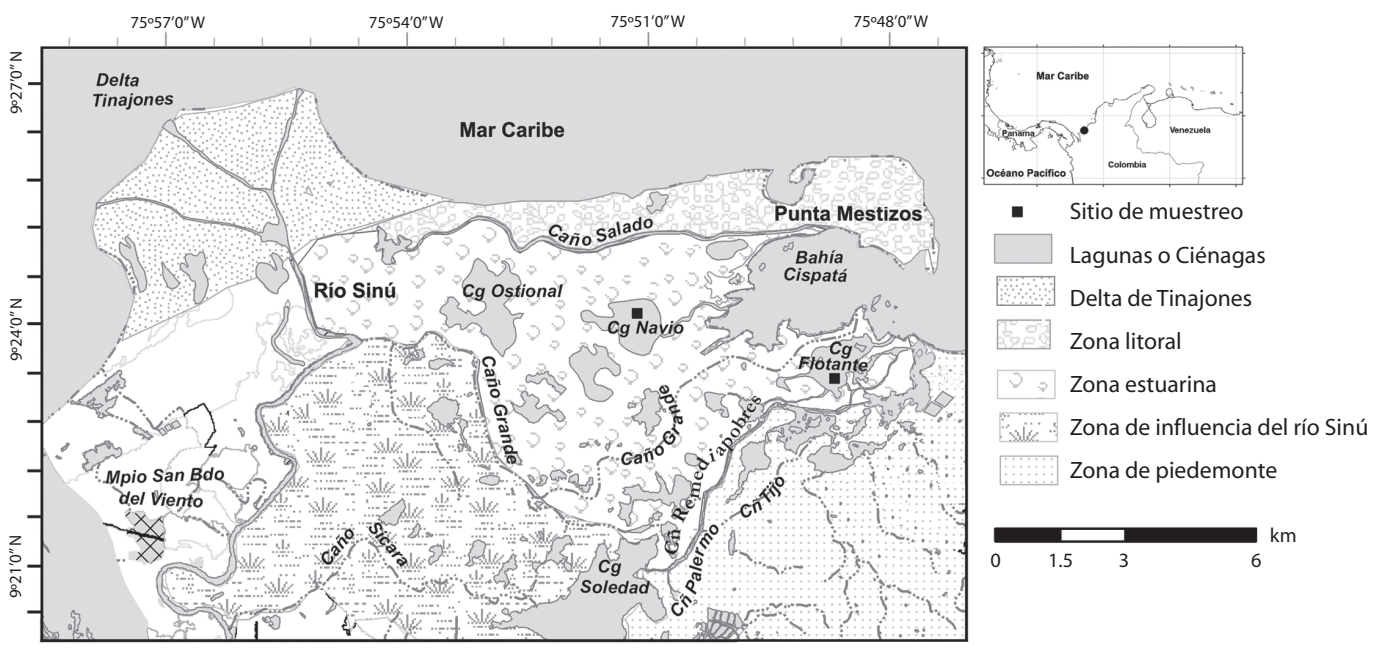

Fig. 1. Zona de estudio. Lagunas muestreadas, unidades geomorfológicas y de vegetación descritas en el complejo lagunar Cispatá por Gil-Torres \& Ulloa-Delgado (2001).

Fig. 1. Study area. Geomorphological and vegetation units described for the Cispatá lagoon system by Gil-Torres \& UlloaDelgado (2001). Sampled lagoons are indicated. 
y noviembre se presenta la temporada lluviosa con $230 \mathrm{~mm} / \mathrm{mes}$ (IDEAM 2001). La temperatura media anual es de $27^{\circ} \mathrm{C}$ (Palacio \& Restrepo 1999) y la evaporación potencial anual promedio es de $1826 \mathrm{~mm}$ (Sánchez-Páez et al. 2004).

Vegetación Actual: En la parte alta de la cuenca del río Sinú la vegetación se caracteriza por la presencia de bosque pluvial Premontano (Neotrópicos 1997). En la zona media y baja de la cuenca la mayoría de los bosques han sido reemplazados por pastos con fines de pastoreo y áreas de cultivo de algodón, maíz, sandía y arroz. En la parte baja de la cuenca en el delta de Tinajones y en el complejo lagunar Cispatá, se presentan los bosques de manglar (CVS 2004). Las comunidades rurales anexas al complejo lagunar, obtienen de allí madera para leña y postes, corteza para taninos, pesca artesanal, caza, ecoturismo, recolección de moluscos y crustáceos (ASPROCIG, 2006). Los manglares se han clasificado en cuatro zonas (Gil-Torres \& Ulloa-Delgado 2001) según la geomorfología (Fig. 1):

(i) Zona litoral: Se encuentra entre la línea de costa y las riberas del caño Salado, desde Tinajones hasta punta Mestizos. En esta zona los aportes de agua dulce provienen del río Sinú a través de caños en la época de invierno (Olaya et al. 1991). Se presentan gramíneas (Poaceae) de los géneros Brachiaria, Eragostis y Sporobolus, y árboles como Croton sp, Hibiscus tiliaceus L., Hippomane mancinella L., Thespesia populnea (L.) Sol. ex Correa y Chrysobalanus icaco L., entre otras. Entre las especies rastreras se encuentran Batis maritima L., Melochia crenata Bertero ex Spreng., Salicornia fruticosa L., Spartina sp., Ipomoea pescaprae (L.) R. Br. y Sesuvium portulacastrum (L.) L., asociadas con las especies de manglar Rhizophora mangle L., Laguncularia racemosa (L.) C.F. Gaertn., Avicennia germinans (L.) L., Conocarpus erectus L. y Pelliciera rhizophorae Planch. \& Triana (Sánchez -Páez et al. 2003).

(ii) Zona estuarina: Está ubicada entre el caño Salado hacia el norte, el caño Grande al sur y los caños Tijó, Palermo y Remediapobres al suroriente. Es un sistema de ciénagas marinofluvial con una profundidad en lámina de agua que varía entre 0.5 y $14 \mathrm{~m}$ (Ulloa-Delgado et al. 1998). Comprende los manglares de la parte interna del complejo lagunar con presencia de $R$. mangle, A. germinans, L. racemosa, P. rhizophorae, C. erecta y el helecho Acrostichum aureum L. en masas dispersas (Sánchez-Páez et al. 2004).

(iii) Zona de influencia aluvial: Área ubicada entre el caño Grande al norte y el caño Sicará al sur; al occidente y suroriente, el río Sinú y la ciénaga Soledad. Conformada por especies de vegetación de marismas, tales como Typha dominguensis Pers., Mikania cordifolia (L. f.) Willd., Ambrosia sp., Ludwigia peploides (Kunth) P.H. Raven, Cyperus giganteus Vahl y algunos bejucos Rhabdadenia biflora (Jacq.) Müll. Arg., Sarcostemma sp., Ipomoea setifera Poir. y Solanum scabrum Mill. (Gil-Torres \& Ulloa-Delgado 2001).

(iv) Zona de piedemonte: Comprende la parte suroriental de la zona estuarina influenciada por los caños Tijó, Palermo y Remediapobres hasta la zona de colinas al suroriente. Se presentan relictos de bosques secundarios, que sirven de cercas vivas y sombrío para el ganado, donde se destacan especies como: Pouteria caimito (Ruíz \& Pav.) Radlk., Guazuma ulmifolia Lam., Ficus sp, Astronium graveolens Jacq., Anacardium excelsum (Kunth) Skeels, Spondias mombin L., Ceiba pentandra (L.) Gaertn., Hura crepitans L., Nectandra concinna Nees, Lecythis minor Jacq., Chamaefistula fruticosa (Mill.) Pittier, Machaerium capote Triana ex Dugand y Lonchocarpus sanctae-marthae Pittier (Gil-Torres \& Ulloa-Delgado 2001).

Muestreo de Campo: De las lagunas La Flotante y Navío (Fig. 1) se extrajeron dos secciones de sedimentos de $440 \mathrm{~cm}$ y $100 \mathrm{~cm}$ de longitud, respectivamente. Se utilizó una sonda rusa modificada tipo Macaulay (Traverse 1988). En cada sección se hizo la descripción estratigráfica en términos de color con tabla Munsell, textura al tacto y presencia de restos orgánicos y calcáreos. 
Procesamiento y análisis de datos: $\mathrm{La}$ cronología de las secciones de sedimentos se estableció con base en dataciones con ${ }^{210} \mathrm{~Pb}$ y deducciones a partir de la granulometría y la tasa de acumulación de polen. En el núcleo $\mathrm{La}$ Flotante se hicieron 12 dataciones de ${ }^{210} \mathrm{~Pb}$ en el laboratorio My Core Scientific Inc. En el núcleo Navío se contó con la información correspondiente a 14 datos preliminares no publicados medidos en el laboratorio de Geoquímica y Contaminación Costera del Instituto de Ciencias del Mar y Limnología de la Universidad Autónoma de México, Unidad Mazatlán. Para ambos núcleos se utilizó el modelo CRS (Constant Rate Supply, Appleby \& Oldfield 1983) para calcular la edad de las muestras discretas que fueron utilizadas (cuatro en La Flotante, dos en Navío). En la parte superior de los dos núcleos, donde se obtuvieron fechas de $\mathrm{Pb}$, se utilizó la tasa de acumulación de polen para construir el modelo de edad según el método propuesto por Traverse (1988). Para la sección profunda del núcleo La Flotante se estimó una fecha en la base a partir del cambio en la granulometría de lodos a arenas, teniendo en cuenta la granulometría actual del Golfo de Morrosquillo (Molina et al. 1994) y el modelo de evolución deltaica de Serrano (2001). Entre esta fecha y la última de $\mathrm{Pb}$ se hizo una interpolación lineal. Para la sección profunda del núcleo Navío se extrapoló el modelo superficial.

En la sección de La Flotante se extrajeron 50 muestras en los primeros $200 \mathrm{~cm}$. Cada muestra se obtuvo al concentrar $4 \mathrm{~cm}^{3}$, correspondientes a $4 \mathrm{~cm}$ de profundidad. A partir de $200 \mathrm{~cm}$ y por la baja concentración de polen, se analizaron 13 muestras a intervalos irregulares entre 220 y $440 \mathrm{~cm}$. En la sección Navío se analizaron 20 muestras. Cada muestra se obtuvo al concentrar $2 \mathrm{~cm}^{3}$ por cada centímetro de profundidad en los primeros $20 \mathrm{~cm}$ de la sección. Entre 20 y $100 \mathrm{~cm}$ se analizaron 20 muestras a intervalos de $4 \mathrm{~cm}$.

En el laboratorio, se utilizaron técnicas estándar de preparación de polen (Faegri e Iversen 1989). A cada muestra se le adicionó una tableta de esporas de Lycopodium para calcular la concentración $\left(\right.$ granos $/ \mathrm{cm}^{3}$ ) y la tasa de acumulación de polen (Traverse 1988). Los residuos fueron montados en placas fijas con gelatina glicerinada y analizados a 400x y 1000x con un microscopio de luz transmitida. En cada muestra se contaron entre 250 y 300 granos de polen. Las zonas de polen fueron definidas con base en un análisis cluster realizado mediante el sotfware CONISS (Grimm 1987) y el ordenamiento resultante de un Análisis de correspondencia sin tendencias o DCA (Detrended Correspondence Analisis), en este análisis se incluyeron los táxones con abundancia relativa $>2 \%$ y se utilizó CANOCO (Ter Braak \& Smilauer 2002).

En la sección de La Flotante se hicieron análisis de Carbono total, Nitrógeno y $\mathrm{CaCO}_{3}$ en 22 muestras tomadas cada $4 \mathrm{~cm}$, en los $88 \mathrm{~cm}$ superiores. En la sección Navío estos parámetros se midieron en los $56 \mathrm{~cm}$ superiores, cada centímetro entre 3 y $20 \mathrm{~cm}$ y luego cada $4 \mathrm{~cm}$. $\mathrm{El} \mathrm{C}$ y $\mathrm{N}$ fueron medidos en un analizador elemental del laboratorio de Combustión y Combustibles de la Universidad del Valle, siguiendo la norma ASTM D5373-02. Los carbonatos fueron medidos por medio de digestión en $\mathrm{HCl}$ en un calcímetro tipo Bernard (Quiceno 2008). El Carbono orgánico (Co) fue estimado como la diferencia entre el Carbono total y el Carbono inorgánico $(\mathrm{Ci})$ calculado a partir de los carbonatos. Asimismo, se estimó la razón $\mathrm{Co} / \mathrm{N}$, la cual se ha considerado un indicador de la fuente de materia orgánica, marina vs. fluvial (Lamb et al. 2006). Se hicieron gráficas comparativas en el tiempo para los parámetros geoquímicos de los dos núcleos y análisis de correlación por medio de una matriz de correlación teniendo en cuenta la significancia estadística.

\section{RESULTADOS}

Descripción física de los núcleos: En la base de La Flotante $(440-419 \mathrm{~cm})$ los sedimentos corresponden a arenas limosas de color negro $(5 Y 2.5 / \mathrm{L})$. Entre 419 y $26 \mathrm{~cm}$ son limos arcillosos con cambios de color y contenido de restos orgánicos y carbonatos. Son grises $(5 \mathrm{Y}$ $3 / 1$ ) entre 419 y $300 \mathrm{~cm}$, cambian a verde oliva (5Y $3 / 2)$ entre 300 y $240 \mathrm{~cm}$ y retornan al gris 


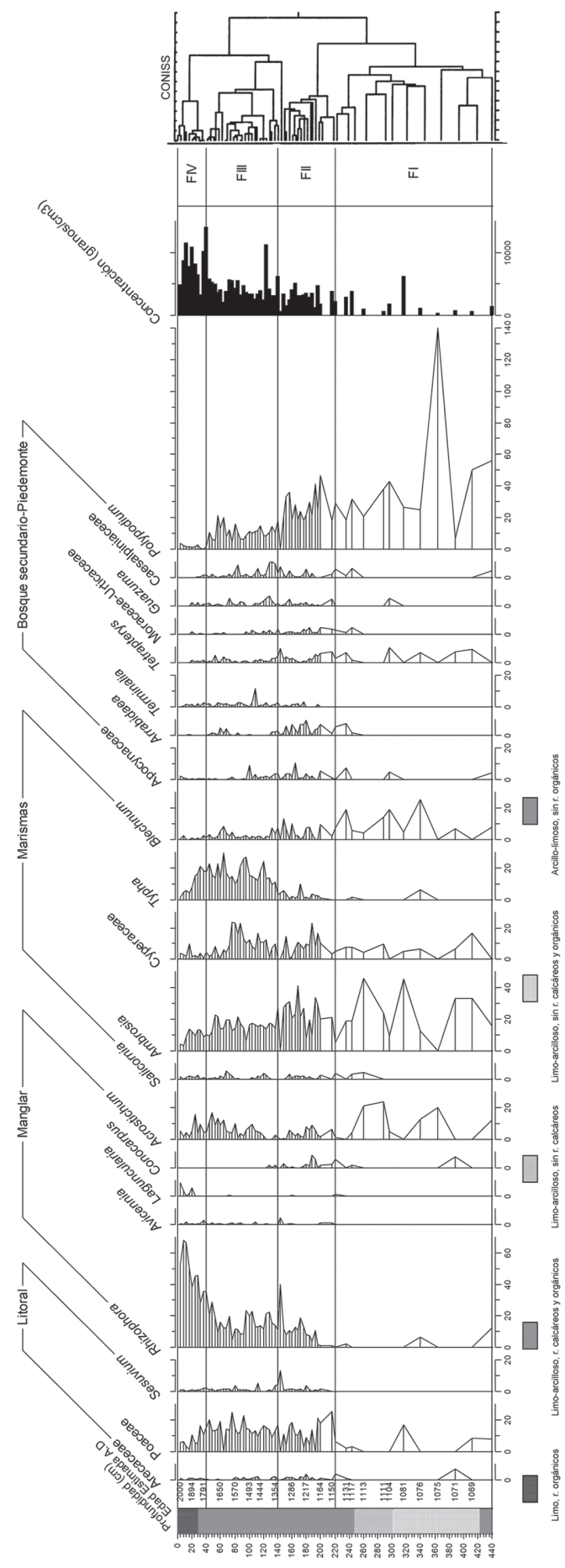

Fig. 2. Polen de la sección La Flotante. Profundidad (cm), edad estimada (A.D.), porcentaje de especies con abundancias relativas $\geq 5 \%$, concentración de polen
(5Y 3/1) con restos orgánicos y calcáreos entre 240 y $26 \mathrm{~cm}$. Hacia el tope de la secuencia, desde 26 a $0 \mathrm{~cm}$, los sedimentos corresponden a limos con restos orgánicos (Fig. 2). En la laguna Navío, los sedimentos corresponden a arcillas-limosas con grados variables en el contenido de restos orgánicos y calcáreos (Fig. 3). No se observaron laminaciones en los núcleos.

Dataciones: Las mediciones de $210 \mathrm{~Pb}$ de ambos núcleos mostraron un perfil decreciente exponencial característico del decaimiento del ${ }^{210} \mathrm{~Pb}$ no soportado en profundidad. De estas se consideraron únicamente fechas asignadas a las muestras que contenían exceso de ${ }^{210} \mathrm{~Pb}(4$ muestras superiores para La Flotante y 2 para Navío, Cuadro 1).

Descripción e interpretación de los diagramas de polen: Los diagramas de porcentajes y tasas de acumulación de polen (Figs. 2 y 3) registran la historia de la vegetación en el complejo lagunar Cispatá durante los últimos 941 años para laguna La Flotante y los últimos 260 años para laguna Navío. Las zonas en las cuales se dividieron los diagramas se definieron con base en los grupos obtenidos con los análisis de Cluster y el análisis de correspondencia (DCA), los cuales fueron coincidentes.

\section{Laguna La Flotante}

Zona FI (440-220cm, 1064-1142 A.D.): En esta zona se obtuvieron bajas concentraciones promedio de polen (1954granos/ $\mathrm{cm}^{3}$ ). El espectro polínico (Fig. 2) fue dominado por esporas de helechos principalmente: Polypodium (6-58\%), Acrostichum $(<17 \%)$ y Blechnum $(<20 \%)$ y vegetación de marismas

$\left(\right.$ granos $/ \mathrm{cm}^{3}$ ) y zonas de polen identificadas con el análisis de correspondencia (DCA) y Coniss.

Fig. 2. Pollen diagram the La Flotante core. From left to right, depth (cm), estimated age (A.D.), pollen percentages of taxa with relative abundances higher than $5 \%$, pollen concentration (grains $/ \mathrm{cm}^{3}$ ) and pollen zones identified by DCA, are shown. 


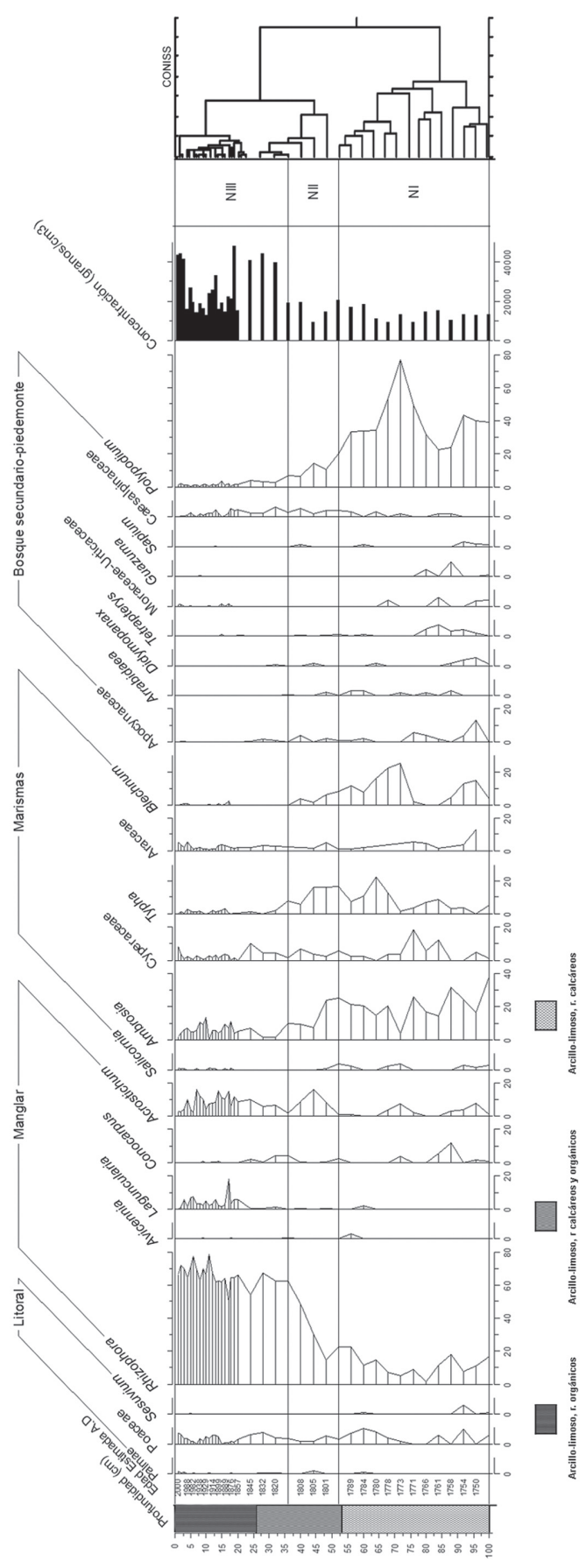

Fig. 3. Polen de la sección Navío. Profundidad (cm), edad estimada (A.D.), porcentaje de especies con abundancias relativas $\geq 5 \%$, concentración de polen (granos $/ \mathrm{cm}^{3}$ ) y zonas de polen identificadas con el análisis de correspondencia (DCA) y Coniss.
Ambrosia (47\%) y Cyperaceae $(<15 \%)$. Otros elementos presentes correspondieron a vegetación de litoral, principalmente Poaceae (17\%). Las bajas concentraciones de polen y la dominancia de algunos helechos evidencian la prevalencia de condiciones marinas sobre las continentales.

Zona FII (220-140cm, 1142-1331 A.D): En esta zona las concentraciones promedio de polen fueron de $3255 \mathrm{granos} / \mathrm{cm}^{3}$. Fue dominada por vegetación de marismas especialmente Ambrosia (32\%), Cyperaceae (17\%) y Typha (9\%) que incrementaron al finalizar la zona. La vegetación de manglar, principalmente, Rhizophora (5\%-19\%), mostró un incremento a partir de la mitad de la zona, mientras que Acrostichum mostró una disminución (6\%$1 \%)$. Los helechos Polypodium y Blechnum disminuyeron respecto a la zona anterior y Poaceae alcanzó valores de $17 \%$. Representa el inicio de la colonización del manglar en un ambiente de marismas facilitado por el aporte de sedimentos aluviales en los alrededores del delta activo en ese periodo de tiempo.

Zona FIII (140-40 cm, de 1331-1738 A.D):

En esta zona las concentraciones promedio de polen fueron de $4343 \mathrm{granos} / \mathrm{cm}^{3}$. Mientras que la vegetación de manglar mostró una tendencia de incremento principalmente Rhizophora (11\%-34\%) y Acrostichum (1\%-9\%), la vegetación de marismas continuó dominando, Typha (26\%), Cyperaceae (22\%) y Poaceae (15\%) se incrementaron, pero Ambrosia disminuyó (15\%). Representa todavía la dominancia de marismas sobre el manglar, es una zona de intercambio entre aguas continentales y oceánicas, con mayor influencia de aguas salobres evidenciada por estas especies de marismas.

Zona FIV (40-0cm, 1738-2000 A.D): En esta zona las concentraciones promedio de

Fig. 3. Pollen diagram the Navio core. From left to right, depth $(\mathrm{cm})$, estimated age (A.D.), pollen percentages of taxa with relative abundances higher than $5 \%$, pollen concentration (grains $/ \mathrm{cm}^{3}$ ) and pollen zones identified by DCA, are shown. 
CUADRO 1

Muestras datadas con 210Pb y utilizadas para estimar la edad de las columnas de sedimento de las lagunas La Flotante y Navio

TABLE 1

$210 \mathrm{~Pb}$ dates used in estimation the age scale in La Flotante and Navio lagoon cores

$\begin{array}{ll}\text { Sección } & \text { Número de Laboratorio } \\ \text { La Flotante } & \text { My core } 831 \\ & \text { My core } 832 \\ & \text { My core } 833 \\ & \text { Análisis granulométrico y geomorfológico } \\ \text { Navío } & \text { Lab Gqca 1LN } \\ & \text { Lab Gqca 2LN }\end{array}$

polen fueron de $8609 \mathrm{granos} / \mathrm{cm}^{3}$. Representa el manglar plenamente establecido, principalmente Rhizophora (34-60\%) mostró una tendencia de incremento y Acrostichum (5\%) disminuyó. La vegetación de marismas representada por Typha (18-5\%), Ambrosia (13-4\%) y Cyperaceae $(5-2 \%)$ y de litoral por Poaceae (15-6\%) disminuyeron. El incremento en la concentración de polen coincidente con la formación del delta de Mestizos y de la bahía de Cispatá (1762 A.D.), indican condiciones de menor energía y predominio de las condiciones continentales sobre las marinas, lo cual permitió el establecimiento y expansión del manglar, generado por la progradación del delta, el cual estaba culminando su formación para esta época.

\section{Laguna Navío}

Zona NI (100-52cm, 1746-1795 A.D): En promedio la zona presentó una concentración de polen de 14113 granos $/ \mathrm{cm}^{3}$. Estuvo dominada (Fig. 3) por los helechos Polypodium (25\%) y Blechnum (14\%), vegetación de marismas representada por Ambrosia (22\%) y Typha $(17 \%)$, las cuales alternaron sus picos de dominancia, reflejando la variabilidad en la influencia mareal y las etapas iniciales de desarrollo del manglar dominado por Rhizophora (25\%),

$\begin{array}{ccc}\text { Profundidad }(\mathrm{cm}) & \text { Edad }{ }^{210} \mathrm{~Pb} & \text { A.D } \\ 0-3 & 5 & 2000 \\ 7-9 & 18 & 1987 \\ 13-14 & 42 & 1963 \\ 419-440 & & 1064 \\ 0-1 & 5 & 2000 \\ 7-8 & 67 & 1938\end{array}$

con un comportamiento creciente hacia el final de la zona.

Zona NII (52-36cm, 1795-1814 A.D): La concentración de polen promedio fue de 16162 granos $/ \mathrm{cm}^{3}$. Esta zona representó la transición hacia el establecimiento del manglar, se caracterizó por la dominancia de Rhizophora, que alcanzó valores de $63 \%$ y por la disminución de la vegetación de marismas, representada principalmente por cambios en Ambrosia (27\%-10\%) y Typha (18\%-7\%) que disminuyeron hacia el final. Este cambio registra el incremento de aportes de sedimentos continentales.

Zona NIII (32-0cm, 1814-2000 A.D): Representa el manglar plenamente establecido. Los porcentajes de Rhizophora alcanzaron $65 \%$. Se encontró Laguncularia $(4 \%)$ hacia la mitad de la zona, lo cual se relaciona con la predominancia de condiciones de salinidad medias a bajas y la perturbación antrópica reciente. La mayoría de los táxones de vegetación de marismas y de piedemonte presentaron disminución en esta zona.

Análisis de Correspondencia: En las Figuras 4A y 4B se presentan los cuatro grupos identificados en el DCA del registro de $\mathrm{La}$ Flotante para los táxones polínicos (4A) y las profundidades a las que se tomaron las muestras (4B), los cuales coinciden con los grupos identificados por análisis de cluster, indicados 


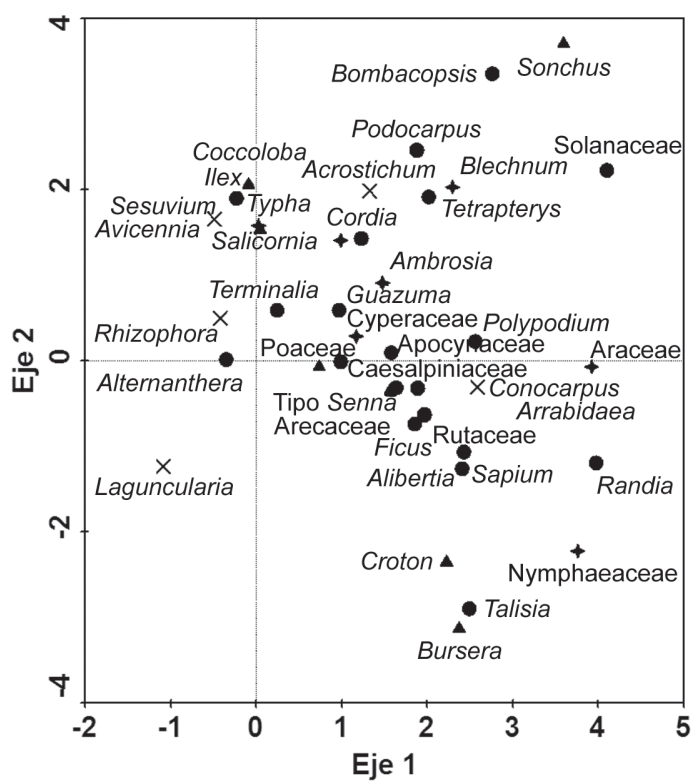

A

- Litoral

+ Marismas

$\times$ Manglar

- Bosque secundario-Piedemonte

B

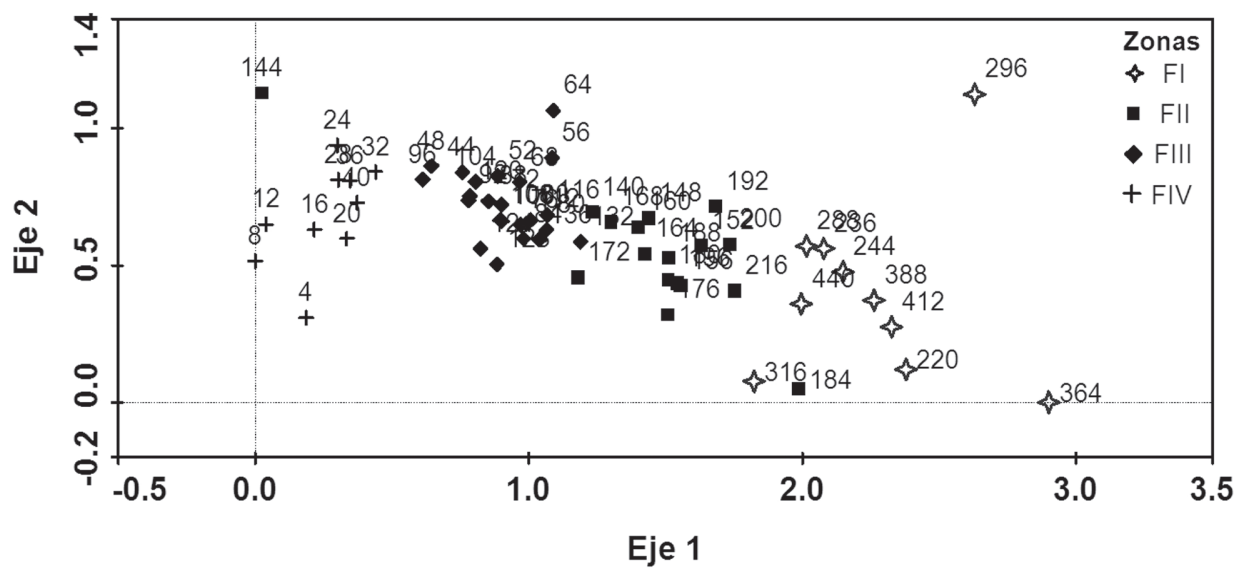

Fig. 4. Diagramas de ordenación del análisis de correspondencia (DCA), para la sección de laguna La Flotante. A). Dispersión de los taxones mayores de 5\%. $\mathbf{\Delta}$ Vegetación de litoral, $\diamond$ Marismas, $\mathrm{x}$ Manglar, $\bullet$ Bosque secundarioPiedemonte. B). Agrupamiento de las muestras (profundidad en cm), $\diamond$ FI (440-220 cm), $\square$ FII (216-140 cm), $\diamond$ FIII (136-44 cm) y + FIV (40-0).

Fig. 4. Ordination diagrams of Detrended Correspondence Analysis (DCA) of the La Flotante lagoon. A). Biplot of taxa with percentages higher than 5\%. \%. $\boldsymbol{\Delta}$ Littoral vegetation, $\diamond$ Saltmarshes, $\mathrm{x}$ Mangoves, $\bullet$ Secondary forest-Foothills. B) Biplot

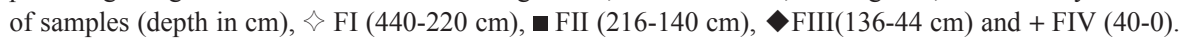


en el diagrama de polen. El valor propio (eigenvalue) alcanzado por el primer eje fue de 0.37 y por el segundo de 0.10 , conjuntamente estos dos ejes explicaron el $32.3 \%$ de la variación en los datos.

La figura 4A muestra la dispersión de los táxones polínicos. En ésta la vegetación de manglar se ubica al lado izquierdo del segundo eje, mientras que la de marismas hacia el extremo derecho. Los taxones correspondientes a la vegetación de piedemonte se encuentran hacia el centro de la gráfica. En el mismo sentido, la figura 4B muestra los cuatro grupos, producto del ordenamiento de las muestras en los dos primeros ejes, los cuales se relacionan con la profundidad y revelan fases de cambio de la vegetación. El primer grupo incluye las muestras ubicadas entre 440 y $220 \mathrm{~cm}$ y caracteriza la fase dominada por helechos y vegetación de marismas. El segundo, entre 220 y $140 \mathrm{~cm}$ y el tercero, entre 140 y $40 \mathrm{~cm}$, representan las fases iniciales de colonización del manglar, en las cuales esta vegetación reemplaza la vegetación de marismas. Y el cuarto agrupa las muestras correspondientes a los $40 \mathrm{~cm}$ superficiales en las cuales se identifica el manglar plenamente establecido.

Los resultados del DCA para la sección Navío se presentan en las Figuras 5A y 5B. Los autovalores del primer y segundo eje fueron de 0.50 y 0.15 , respectivamente. Estos dos ejes explicaron el $42.1 \%$ de la variación en los datos. En la figura 5A, se observa una tendencia de cambio de izquierda a derecha en la ubicación de los táxones; hacia el extremo izquierdo se identifica la vegetación de manglar y hacia el extremo derecho la vegetación de marismas, mientras que en el centro se encuentran helechos y vegetación secundaria registrada en la transición entre manglar y piedemonte.

En el ordenamiento de las muestras en la figura 5B se pueden identificar tres grupos según la profundidad, los cuales coinciden con los grupos identificados por el análisis de cluster, e indicados en el diagrama de polen. El primer grupo lo componen las muestras entre 100 y $52 \mathrm{~cm}$, el segundo entre 52 y $36 \mathrm{~cm}$ y el tercero, las muestras de los $36 \mathrm{~cm}$ superficiales.
Estos grupos representan los cambios de la vegetación, desde marismas, transición a manglar, y manglar plenamente establecido, respectivamente.

Análisis geoquímicos: En las Figuras 6 y 7 se presentan las curvas de carbono orgánico $(\% \mathrm{Co})$, carbono inorgánico $(\% \mathrm{Ci})$, la relación $\mathrm{C} / \mathrm{N}$, el porcentaje de carbonatos $\left(\% \mathrm{CaCO}_{3}\right)$, la tasa de sedimentación y el porcentaje de polen de Rhizophora en las secciones estudiadas.

Los registros geoquímicos cubrieron desde 1607 A.D. hasta el presente para la ciénaga La Flotante y desde 1789 A.D. para la ciénaga Navío. Los porcentajes de carbono orgánico e inorgánico fueron más altos en Navío (4 y $3 \%$, respectivamente) que en La Flotante (1.1 y $1.4 \%$ ). La razón $\mathrm{C} / \mathrm{N}$ fue también mayor en Navío (máximo de 15.5 respecto a un máximo de 6.8 en La Flotante). Asimismo, las tasas de sedimentación en la laguna Navío fueron prácticamente el doble que en la Flotante (valores medios de 0.5 y $0.25 \mathrm{~cm} / \mathrm{año}$, respectivamente). Hacia 1679 A.D. se presenta una ligera disminución de la razón $\mathrm{C} / \mathrm{N}$ y un ligero aumento de $\mathrm{Ci}$ en la ciénaga La Flotante. Estas variaciones no son significativas si se tiene en cuenta la precisión del método de medición de los carbonatos. En 1894 A.D. se presentó en esta sección un aumento importante en el $\mathrm{Ci}$, a la vez que disminuyeron el Co y la razón $\mathrm{C} / \mathrm{N}$. Este mismo patrón se encontró en la sección de Navío alrededor de 1910. En Navío hay otra disminución en Co y $\mathrm{C} / \mathrm{N}$ alrededor de 1835 que no coinciden con un máximo en $\mathrm{Ci}$ y que en la sección de La Flotante se percibe de forma no significativa alrededor de 1840 . En Navío, los valores de $\mathrm{C} / \mathrm{N}$ alcanzan valores de 10 o mayores en dos períodos: de 1814 a 1835 A.D. y de 1860 a 1890 A.D. En la laguna La Flotante las variables Ci, Co, C/N, y Rhizophora presentaron correlación negativa significativa $(\mathrm{p}<0.05)$ con la profundidad. También la correlación entre Co y C/N con Rhizophora fue altamente significativa $(\mathrm{p}<0.01)$. En Navío, la variable edad tuvo correlación significativa $(\mathrm{p}<0.01)$ negativa con Rhizophora, pero con la sedimentación presentó correlación positiva 

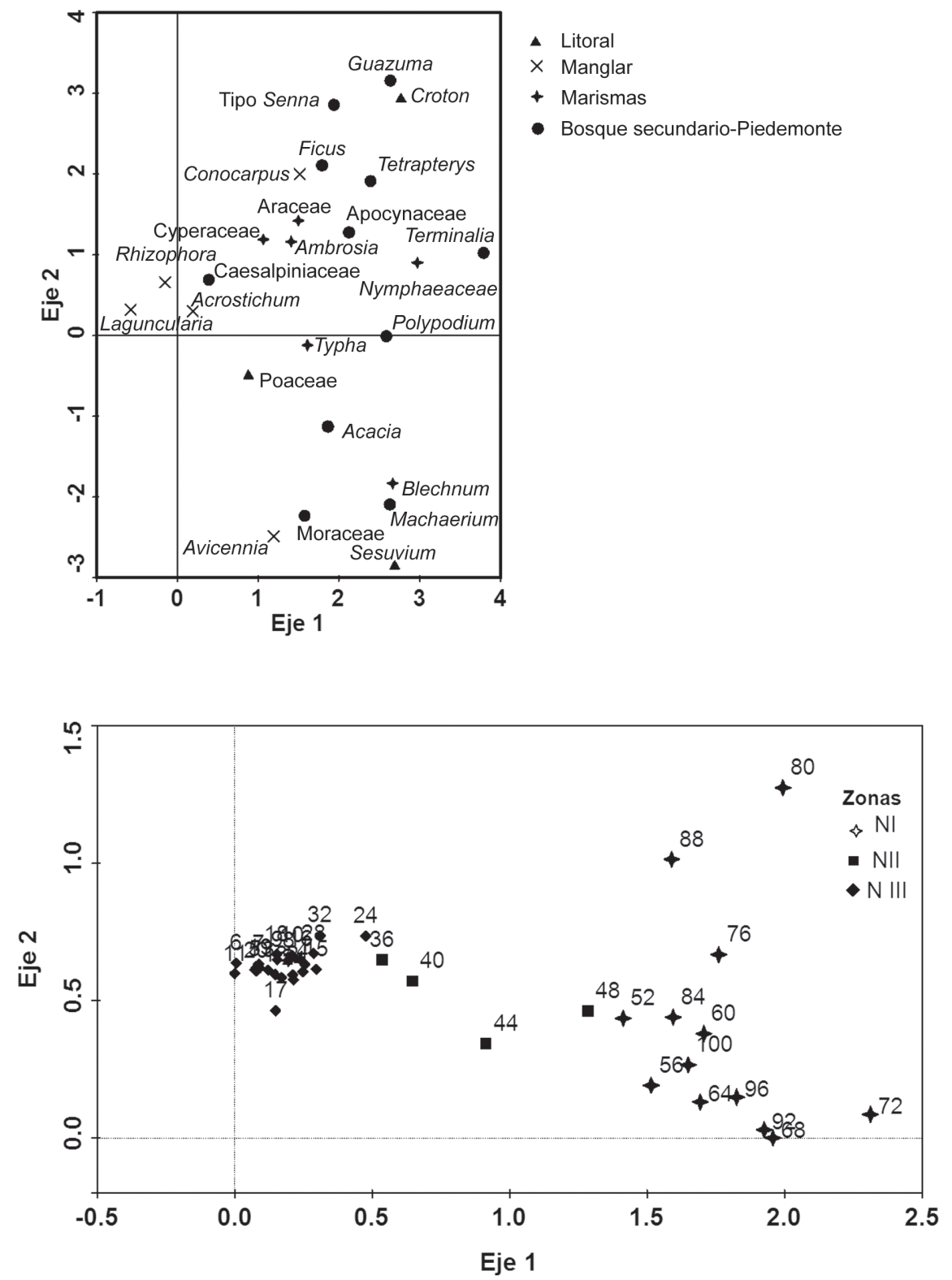

Fig. 5. Diagramas de ordenación del análisis de correspondencia (DCA), para la sección de la laguna Navío. A) Dispersión de los taxones mayores de 5\%, $\boldsymbol{\Delta}$ Vegetación de litoral, $\diamond$ Marismas, x Manglar, $\bullet$ Bosque secundario-Piedemonte. B) Agrupamiento de las muestras (profundidad en cm), $\diamond$ NI $(100-52 \mathrm{~cm}), \mathbf{N I I}(40-36 \mathrm{~cm})$ y $\diamond$ NIII $(32-0 \mathrm{~cm})$.

Fig. 5. Ordination diagrams of Detrended Correspondence Analysis (DCA) of the Navio lagoon A). Biplot of taxa with percentages higher than $5 \%, \boldsymbol{\Delta}$ Littoral vegetation, $\diamond$ Saltmarshes, $x$ Mangoves, $\bullet$ Secondary forest-Foothills. B) Biplot of samples (depth in cm), $\diamond$ NI $(100-52 \mathrm{~cm}), \mathbf{N I I}(40-36 \mathrm{~cm})$ and $\diamond$ NIII $(32-0 \mathrm{~cm})$. 

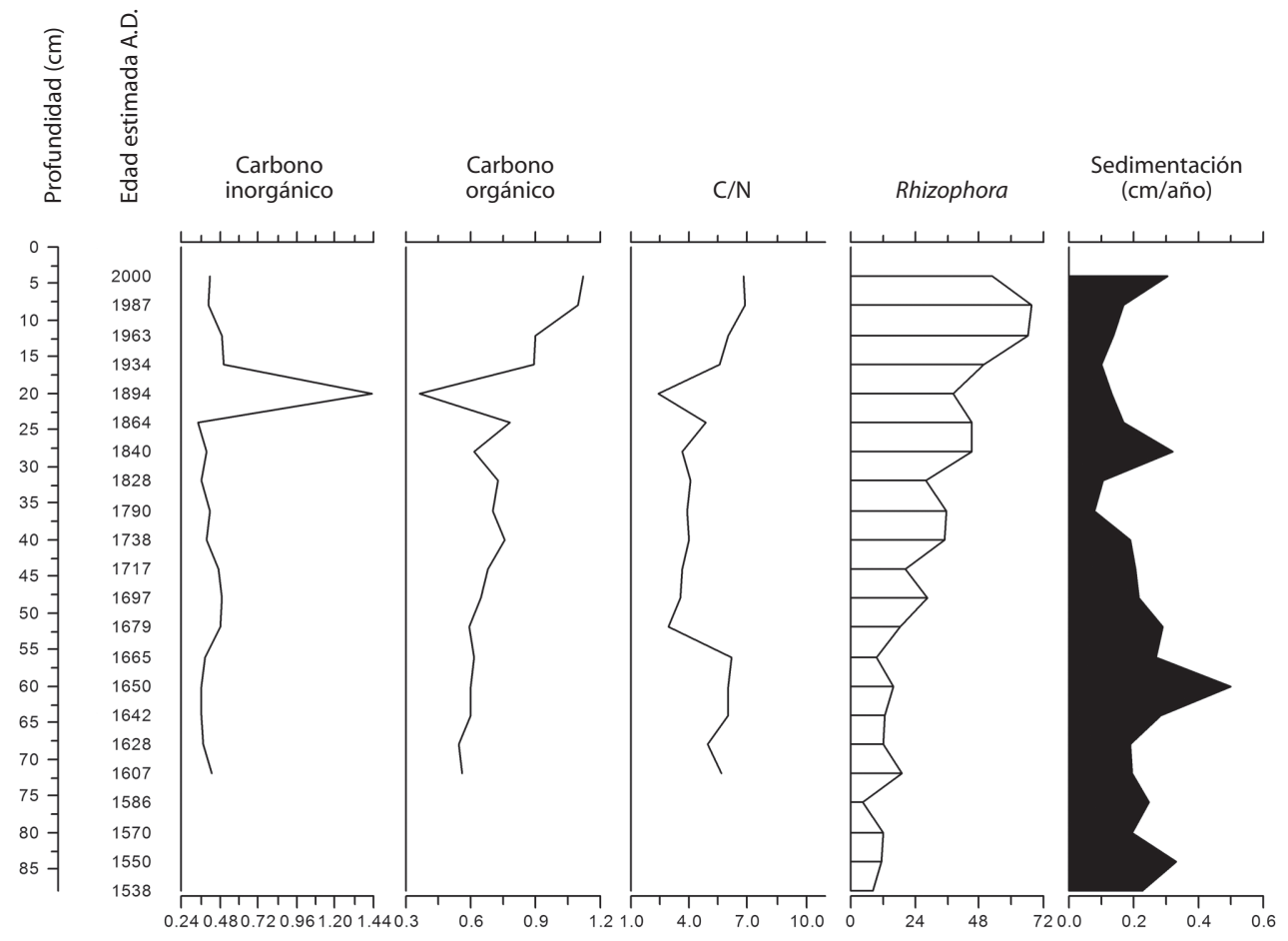

Fig. 6. Cambios de Carbono inorgánico (\%Ci), Carbono orgánico (\% Co), relación Carbono Nitrógeno $(\mathrm{C} / \mathrm{N})$, porcentaje de Carbonatos $\left(\% \mathrm{CaCO}_{3}\right), \%$ de Rhizophora y logaritmo de la tasa de sedimentación ( $\mathrm{cm} /$ año), a lo largo de la profundidad (cm) y edad estimada (A.D.) en la sección de la laguna La Flotante.

Fig. 6. Diagram showing changes in inorganic Carbon $(\% \mathrm{Ci})$, organic Carbon $(\% \mathrm{Co})$, Carbon/Nitrogen ratio $(\mathrm{C} / \mathrm{N})$, percentage of Carbonates $\left(\% \mathrm{CaCO}_{3}\right)$, pollen percentage of Rhizophora and logarithm of the sedimentation rate $(\mathrm{cm} / \mathrm{yr})$, along depth $(\mathrm{cm})$ and estimated age (A.D.) in La Flotante lagoon core.

significativa $(\mathrm{p}<0.01)$; de igual manera Co, $\mathrm{C} / \mathrm{N}$ y Rhizophora tuvieron una correlación significativa $(\mathrm{p}<0.01)$

\section{DISCUSIÓN}

Los modelos cronológicos utilizados mostraron ser consistentes con los cambios ocurridos en el delta y la vegetación. Especialmente la correspondencia entre el aumento del porcentaje de polen de manglar en la sección de la Flotante y la disminución de condiciones fluviales en el sistema, a partir de 1938 cuando se formó el delta actual (Tinajones). Los valores máximos de carbonatos y mínimos de Co registrados alrededor de 1900 A.D., en ambos núcleos, permiten correlacionar las dos secuencias. Las fechas asignadas para este evento tienen diferencias menores a 15 años entre ambas secciones. El evento de mínimo Co ocurrido alrededor de 1837 presenta diferencias de 5 años entre éstas. Las diferencias entre los dos núcleos, considerando la fracción superior de ambos, se pueden resumir en: mayores concentraciones de polen, tasas de sedimentación y materia orgánica para Navío, la cual tiene una mayor influencia aluvial. Asimismo, la razón $\mathrm{C} / \mathrm{N}$ en la Flotante presenta valores entre 4 y 6 , que indican el origen marino de la materia orgánica, al menos desde 1600 A.D. Por otro lado, en la ciénaga Navío la razón C/N muestra un cambio en el origen de la materia orgánica, 


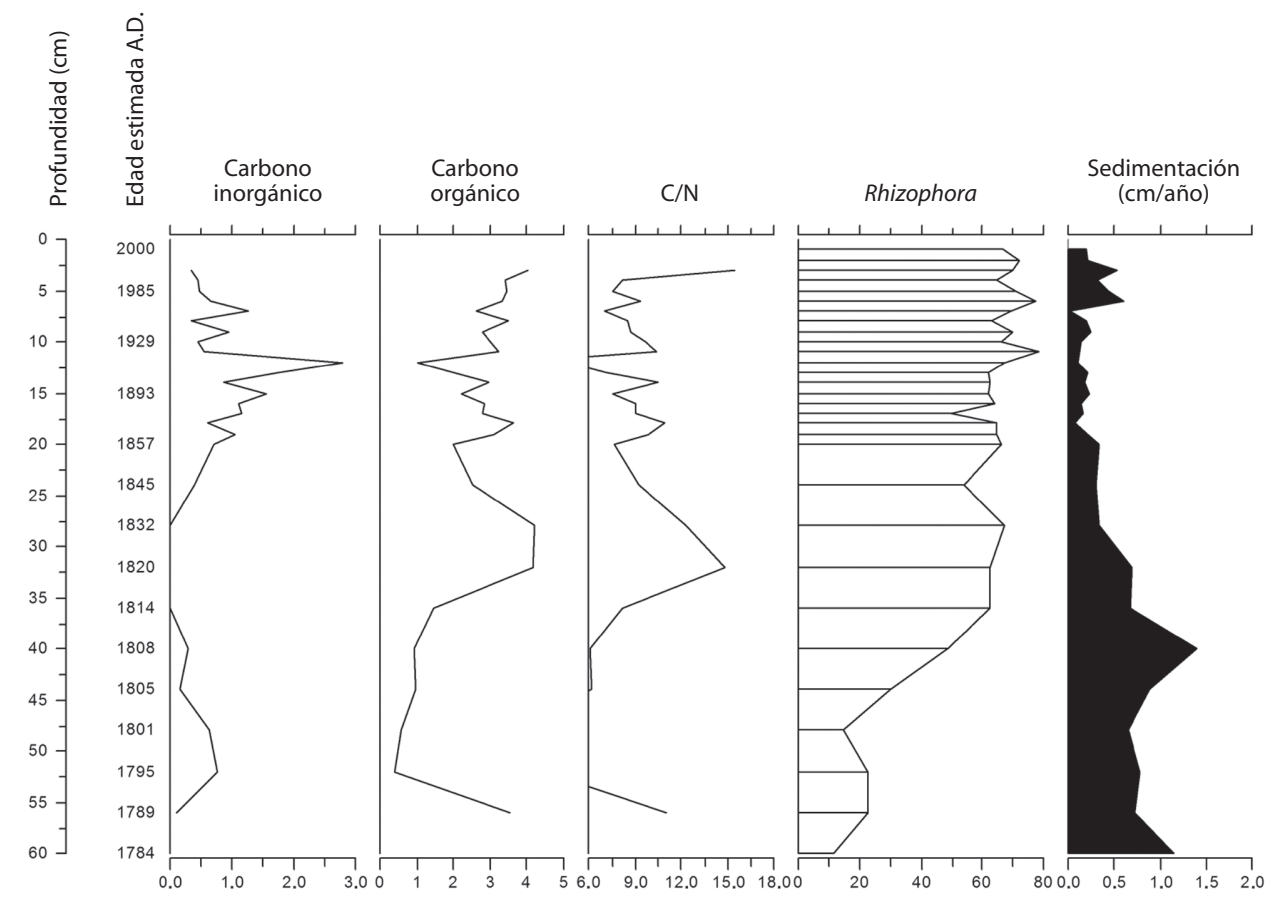

Fig. 7. Cambios de Carbono inorgánico (\%Ci), Carbono orgánico $(\% \mathrm{Co})$, relación Carbono Nitrógeno $(\mathrm{C} / \mathrm{N})$, porcentaje de Carbonatos $\left(\% \mathrm{CaCO}_{3}\right), \%$ de Rhizophora y logaritmo de la tasa de sedimentación ( $\left.\mathrm{cm} / \mathrm{año}\right)$, a lo largo de la profundidad (cm) y edad estimada (A.D.) en la sección de la laguna Navío.

Fig. 7. Diagram showing changes in inorganic Carbon $(\% \mathrm{Ci})$, organic Carbon $(\% \mathrm{Co})$, Carbon/Nitrogen ratio $(\mathrm{C} / \mathrm{N})$, percentage of Carbonates $\left(\% \mathrm{CaCO}_{3}\right)$, pollen percentage of Rhizophora and logarithm of the sedimentation rate $(\mathrm{cm} / \mathrm{yr})$, along depth (cm) and estimated age (A.D.) in Navio lagoon core.

marino antes de 1805 A.D. y terrestre hasta 1814 A.D.; a partir de esta fecha al presente se registran aportes combinados, con una intrusión marina alrededor de 1900 A.D. Valores menores a 10 indican origen marino para la materia orgánica y mayores a 12 indican origen terrestre; los valores cercanos a 10 indican mezcla de ambos tipos (Andrews et al. 1998, Goñi et al. 2003, Lamb et al. 2006). Las diferencias entre los núcleos indican mayor influencia aluvial en Navío, mientras que La Flotante ha estado más expuesta a procesos marinos, lo cual se relaciona con la ubicación de las lagunas con respecto al cauce principal del río y la línea de costa, respectivamente.
En el periodo comprendido entre 1064 A.D. y 1738 A.D. (zonas FI, FII y FIII) en la sección de La Flotante, las concentraciones de polen fueron menores a 5000 granos $/ \mathrm{cm}^{3}$, sin embargo, están dentro de los valores registrados en ambientes marinos en el noroeste de África (Hooghiemstra et al. 2006). Estos autores estimaron que las bajas concentraciones de polen en sedimentos marinos (entre 10 y $5000 \mathrm{granos} / \mathrm{cm}^{3}$ ) se relacionan, entre otros factores, con altas tasas de oxidación de los granos y el retrajabamiento de los sedimentos por la dinámica fluvio-marina. El análisis estratigráfico de los sedimentos de fondo de La Flotante y los estudios de Molina et al. (1994) en la bahía de Cispatá y el golfo de Morrosquillo, 
cercanos a la zona de estudio (Fig. 1), así como la reconstrucción geomorfológica del delta de río Sinú (Serrano 2001), señalan que antes de 1064 A.D. las condiciones eran predominantemente marinas, el impacto del oleaje y la erosión costera impidieron la acumulación de polen y sedimentos finos, hasta 1762 A.D., cuando empezó la formación de la espiga de Mestizos. Los granos de polen encontrados hasta entonces, probablemente provinieron de la vegetación establecida en los alrededores de los deltas, ya que los porcentajes coinciden con los registrados por Urrego et al. (2009) para polen transportado desde el continente hasta el océano. Los cambios de la vegetación registrados en laguna La Flotante, se pueden resumir en cuatro fases. En la primera (1064-1142 A.D.) la abundancia de esporas de helechos trasportadas grandes distancias por los ríos hacia el mar (Urrego et al. 2009), evidencian la predominancia de las condiciones marinas. Entre 1142 y 1331 A.D. el aporte de sedimentos aluviales facilita la colonización del manglar en los alrededores del delta activo (Robertson \& Martínez 1999, Serrano 2001). En la tercera fase (1331 y 1738 A.D.), permanecen condiciones de marismas con mayor influencia de aguas salobres evidenciada por la presencia de Typha (Glenn et al. 1995), Cyperaceae y Poaceae (Urrego et al. 2009). En la última fase (después de 1738 A.D.) se establece plenamente el manglar. El incremento de la concentración y de los porcentajes de polen de las especies de manglar (Urrego et al. 2009) en La Flotante, a partir de la formación del delta de Mestizos y de la bahía de Cispatá (1762 A.D.), indican predominio de condiciones continentales, de menor energía, lo cual permitió la colonización gradual de la vegetación de manglar, generada por la progradación del delta (Robertson \& Martínez 1999, Serrano 2001). Por su parte, la mayor resolución del núcleo Navío permite detallar la evolución en esta última fase. Entre 1746 y 1795 A.D. la vegetación de marismas y la alternancia en los picos de Ambrosia y Typha reflejan la variabilidad en la influencia mareal, ya que a pesar de ser ambas especies halófilas, parecen no coexistir, ya que Typha soporta mayor salinidad que Ambrosia (Ferreyra 1983, Glenn. et al. 1995, Willard et al. 2001). Igualmente, Blechnum sp., helecho característico de pantanos con baja salinidad, se asocia a las primeras etapas sucesionales del manglar (Mejía \& House 2002, White et al. 2002, González 2003). Para este período la línea de costa estaba muy cerca de allí, la relación $\mathrm{C} / \mathrm{N}$ menor a 7 , indica un origen marino para la materia orgánica (Andrews et al. 1998, Goñi et al. 2003, Lamb et al.2006) y la progradación ocurrida en el delta hacia el lado de la laguna Flotante.

El incremento de la biomasa del manglar en la laguna Navío entre 1795 y 1814 A.D, se evidencia en el incremento del Co, la disminución de $\mathrm{CaCO}_{3}$ y los valores de la razón $\mathrm{C} / \mathrm{N}$ (>10), propios de materia orgánica terrestre. A partir de 1814 A.D., los altos porcentajes de Rhizophora $(70 \%)$ registran manglar plenamente establecido tal como lo señalan estudios que relacionan la vegetación en pie con la lluvia de polen (Engelhart et al. 2007, Urrego et al. 2009). En 1835 la disminución repentina de Co, no asociada con una disminución en $\mathrm{Ci}$, podría indicar un pulso de materiales terrígenos. En 1849 A.D., la desembocadura del río migró hacia la bahía de Cispatá (Robertson \& Martínez 1999), lo cual generó la colmatación de la bahía, producto de mayores aportes fluviales y permitió la consolidación del manglar en el área. Así mismo, la presencia de polen de Laguncularia en esta época indica la salinidad media a baja en los manglares (Medina et al. 1990) y la intervención antrópica (Urrego et al. 2009), ya que para esta época algunas de las áreas cenagosas, drenadas por los caños, se dedicaban al cultivo de arroz y los distributarios a la navegación (Troll \& Schmidt 1985). Entre este período y 1900 A.D. la razón C/N indica predominio de la materia orgánica terrestre en la laguna Navío.

Alrededor de 1900 A.D., se registró una intrusión marina intensa, en ambos núcleos con un aumento significativo de $\mathrm{Ci}$ y una disminución de Co y en la razón C/N. En 1938 A.D. se presentó el proceso de avulsión del río Sinú que dio origen al actual delta de Tinajones. Adicionalmente, los caños distributarios 
Grande, Sicará, Remediapobres y Tijó (Fig. 1) penetraron el cuerpo mareal, lo que ayudó a la formación del complejo lagunar actual, en un área aproximada de $150 \mathrm{~km}^{2}$ (Robertson \& Chaparro 1998, Serrano 2004). En este período se presentaron las mayores proporciones de Rhizophora en ambos núcleos (70\%), por la escasez de agua dulce los cultivos de arroz se malograron y la navegación se hizo imposible en la bahía de Cispatá (Troll \& Schmidt 1985). Sin embargo, el incremento del polen de Laguncularia, desde entonces evidencia la intervención del manglar (Urrego et al. 2009) para la extracción de madera por los pobladores locales (ASPROCIG 2006). Los incrementos en las concentraciones de polen, los cambios en los valores de la relación $\mathrm{C} / \mathrm{N}$ y los porcentajes de $\mathrm{Ci}$ registrados responden a los cambios geomorfológicos (progradación del sistema), al intercambio de materia y energía entre el río y el mar, y parcialmente a la intervención antrópica ocurrida en el sistema lagunar. Aunque las secciones analizadas mostraron diferencias entre ellas con respecto a la influencia marina y fluvial, relacionadas con la distancia a la línea de costa y los procesos de depositación de sedimentos continentales y marinos, presentaron tendencias análogas de establecimiento e incremento de la vegetación de manglar durante los últimos 150 años. Queda por determinar si las condiciones climáticas a largo plazo se reflejan en las variaciones del núcleo.

Cambios a escala regional: El incremento en los porcentajes de polen (especialmente de Rhizophora) y la relación C/N partir de 1861 A.D. reflejan el establecimiento y expansión del manglar en respuesta a los mayores aportes fluviales, a la colmatación de la bahía y al incremento en el nivel del mar indicado por el incremento en las concentraciones de carbonatos $\left(\mathrm{CaCO}_{3}\right)$. Esta tendencia de incremento en las poblaciones de manglar a partir de 1850 , se ha registrado en otros ecosistemas tropicales (Cohen et al. 2005, Carvalho et al. 2006) y se ha asociado con el incremento en el nivel del mar y las precipitaciones (Rull 1999, Polissar et al. 2006, González et al. 2010) hacia el final de la PEH, durante la cual estos fueron menores. Esta expansión de los manglares en Cispatá desde 1850 es el resultado del efecto combinado de los cambios geomorfológicos, que involucran altas tasas de acreción gobernadas por las descargas de sedimentos aluviales y el incremento en el nivel del mar, tal como se ha registrado en otros sitios (Woodroffe 1990, 1999, Parkinson et al. 1994, Snedaker 1995, Alongi 2008). La tendencia global del incremento en el nivel del mar iniciado a partir de 1850 se ha identificado recientemente en el Caribe colombiano (Andrade et al. 2003, Ruíz-Ochoa et al. 2008), con base en la información del mareógrafo ubicado en Cartagena, donde se reporta un aumento en el nivel del mar de $0.6 \mathrm{~cm} / a n ̃ o$, entre 1950 y $1993(25 \mathrm{~cm}$ en 44 años). Las tasas de sedimentación estimadas por Serrano $(2001,2004)$, a partir de la comparación de mapas batimétricos para los intervalos de tiempo considerados en esta investigación (1762-1849 y 1849-1938), son del orden de 5.8 y $3.2 \mathrm{~cm} /$ año, respectivamente, y consideradas por esta autora más bajas de lo esperado, lo cual se explica por procesos de subsidencia y compactación de sedimentos. Las tasas de sedimentación promedio para las lagunas La Flotante y Navío entre 1849 y 1938 fueron menores $(0.13$ y $0.18 \mathrm{~cm} / \mathrm{año})$ y posterior a 1938, se incrementaron a 0.20 y $0.33 \mathrm{~cm} / \mathrm{año}$, respectivamente. Cabe destacar que la estimación que hace Serrano (2001, 2004) considera el área en general y nuestro trabajo exclusivamente las lagunas, las cuales normalmente presentan tasas de sedimentación menores. Aunque estas tasas son inferiores a las del incremento actual del nivel del mar (0.6cm/año) (Andrade et al. 2003, Ruíz-Ochoa et al. 2008), la expansión de los manglares en Cispatá reflejan, en parte, el balance entre este incremento en el nivel del mar y el aporte de sedimentos aluviales en el último período, como condición necesaria para esta expansión (Parkinson et al. 1994).

Se estima que los manglares del Caribe podrían soportar tasas de incremento en el nivel del mar entre $0.08-0.09 \mathrm{~cm} /$ año y estarían en riesgo de desaparición con incrementos entre 
$0.09 \mathrm{~cm} /$ año y $0.12 \mathrm{~cm} /$ año (Ellison \& Stoddart 1991, Ellison 1993, Field 1995). Sin embargo, aún no se conocen con certeza los límites máximos que podrían soportar (Snedaker 1995, Alongi 2008) puesto que los manglares cercanos a Key West, en La Florida, han migrado hasta $1.5 \mathrm{~km}$ hacia tierra adentro desde 1940 , con un incremento del nivel del mar entre 0.23 y $0.27 \mathrm{~cm} /$ año (Ross et al. 2000). El IPCC (2007) proyecta que a escala global el incremento promedio del nivel medio del mar podría ser de $88 \mathrm{~cm}$ para el 2100 , lo cual excedería los límites hasta ahora registrados y crea incertidumbre acerca del futuro de los manglares de Cispatá. Su supervivencia dependerá en parte de los aportes de sedimentos aluviales (Parkinson et al. 1994) y de procesos bióticos como el incremento de la masa de raíces lo cual elevaría el nivel del suelo de los manglares (McKee et al. 2007). Es probable que los manglares puedan acoplarse a las nuevas condiciones ambientales y sigan incrementando su extensión como lo han hecho en los últimos 150 años y se presente desplazamiento de la línea de costa mar adentro, ya que las condiciones topográficas y la batimetría de la plataforma continental en esta zona del Caribe son propicias, así como la dinámica progradante del río Sinú, que garantizaría el aporte de sedimentos aluviales que contrarrestarían el aumento en el nivel del mar, dando estabilidad al manglar a largo plazo. Sin embargo, también seguirían registrándose pérdidas por efecto de la erosión que se presenta actualmente en las playas, por la retención de sedimentos y el impacto en los caudales máximos y mínimos de la represa Urrá construida hace 10 años. No obstante, investigaciones futuras deberán enfocarse en la cuantificación y cambios de las tasas de sedimentación en las diferentes geoformas del sistema, por efecto de la evolución del delta del rio Sinú y el impacto en cada una de ellas del incremento actual en el nivel del mar, para determinar si se mantienen las condiciones de equilibrio que garanticen la permanencia del manglar o si este incremento supera el umbral que permita la progradación del manglar, convirtiéndolo en un sistema erosivo mas que progradante.

\section{AGRADECIMIENTOS}

Agradecimientos a la Dirección de Investigaciones de la Universidad Nacional de Colombia-Sede Medellín (DIME), por financiar el proyecto "Registro climático del fenómeno de La Niña en la zona costera del área de influencia del río Sinú, Caribe colombiano", del cual hace parte esta investigación. Al Departamento de Ciencias Forestales, la Maestría en Bosques y Conservación Ambiental y al laboratorio de Ecología César A. Pérez. A Ana Carolina RuizFernández del Intituto de Ciencias del Mar y Limnología de la Universidad Nacional Autónoma de México, por la información preliminar de las fechas $210 \mathrm{~Pb}$ de la Sección de Navío y a María Mercedes Quinceno por sus aportes.

\section{RESUMEN}

En el complejo lagunar de Cispatá se localiza una de las mayores extensiones de manglares del Caribe colombiano. Por medio del análisis de los cambios en la estratigrafía, el registro palinológico, la concentración de calcita y la relación carbono-nitrógeno, en dos secciones de sedimentos, se reconstruyó la dinámica de estos manglares durante los últimos 900 años. Las edades se establecieron con base en dataciones con ${ }^{210} \mathrm{~Pb}$ y análisis granulométrico. El establecimiento y expansión de los manglares, se relacionó con los procesos fluviomarinos que involucraron la formación de las lagunas. Entre 1064 A.D. y 1762 A.D. se formó la espiga Mestizos y predominaron condiciones marinas. La colonización de la vegetación herbácea ocurrió entre 1142 y 1331 A.D. y la de los manglares a partir de 1717 A.D., por la progradación del delta. Desde 1849 A.D., la migración del delta hacia la bahía de Cispatá, el incremento en el nivel del mar y las tasas de acreción de sedimentos aluviales, permitieron la expansión del manglar en el área. A partir de 1900 se registró una intrusión marina en ambas lagunas. A partir de 1938 A.D, la migración del delta a su posición actual dio origen al sistema lagunar y mayor expansión de los manglares.

Palabras clave: manglares, palinología, lagunas costeras, Cispatá, Caribe colombiano

\section{REFERENCIAS}

Alongi, D.M. 2008. Mangrove forests: Resilience, protection from tsunamis and responses to global climatic change. Estuar. Coast. Shelf S. 76: 1-13. 
Andrade, C.A., E.D. Barton \& C.N.K. Mooers. 2003. Evidence for an eastward flow along the Central and South American Caribbean Coast. J. Geophys. Res. 108: 3185-3196.

Andrews, J.E., A.M. Greenaway \& P.F. Dennis. 1998. Combined Carbon Isotope and C/N Ratios as Indicators of Source and Fate of Organic Matter in a Poorly Flushed, Tropical Estuary: Hunts Bay, Kingston Harbour, Jamaica. Estuar. Coast. Shelf S. 46: 743-756.

Appleby, P.G. \& F. Olfield. 1983. The assessment of $\mathrm{Pb}-210$ data from sites with varying sediment accumulation rates. Hydrobiologia 103: 29-35.

ASPROCIG. 2006. Experiencias locales de manejo sostenible de los bosques y de los recursos hídricos. Revista Semillas, 26/27. Grupo Semillas. Bogota Colombia (Consultado 20 enero 2010, www. semillas.org.co/sitio.shtml?apc $=$ e1b-8446059$8446059 \& \mathrm{x}=20154559)$.

Atencio-García, V. 2000. Impactos de la hidroeléctrica Urrá en los peces migratorios del río Sinú. Rev. Temas Agrarios 5: 29-40.

Berger, U., M. Adams., V. Grimm \& H. Hildenbrandt. 2006. Modelling secondary succession of neotropical mangroves: Causes and consequences of growth reduction in pioneer species. Perspec. Plant. Ecol. 7: $243-252$

Blasco, F., P. Saenger \& E. Janodet. 1996. Mangroves as indicators of coastal change. Catena 27: 167-178.

Carvalho do Amaral P.G., M.P. Ledru, F.R. Branco \& P.C.F. Giannini. 2006. Late Holocene development of a mangrove ecosystem in southeastern Brazil (Itanhaém, state of Sao Paulo). Palaeogeogr., Palaeocl., Palaeoecol. 241: 608-620.

Castaño, A.R. 2004. Reconstrucción palinológica de los cambios sucesionales y ambientales del manglar en la baja Guajira en el Holoceno reciente. Tesis de Ingeniería Forestal, Universidad Nacional de Colombia, Medellín, Colombia.

Cohen, M.C.L., H. Behling \& R.J. Lara. 2005. Amazonia mangrove dynamics during the last millennium: The relative sea-level and the Little Ice Age. Rev. Palaeobot. Palyno. 136: 93-108

CVS (Corporación Autónoma Regional de los Valles del Sinú y San Jorge). 2004. Diagnóstico Ambiental de la Cuenca Hidrográfica del Río Sinú. Informe Final. Montería, Colombia.

Díaz, H., J. Suárez \& I. Bustamante. 2000. Determinación de los tipos, clases y cantidades de sedimentos transportados por el río Sinú, y su dinámica, antes de la fase del llenado de Urrá I. Rev. Temas Agrarios 5: 19-32.

Ellison, A.M., B.B. Mukherjee \& A. Karin. 2000. Testing patterns of zonation in mangroves: Scale dependence and environmental correlates in the Sudarbans of Bangladesh. J. Ecol. 88: 561-564.

Ellison, J.C. 1993. Mangrove retreat with rising sea-level, Bermuda. Estuar. Coast. Shelf S. 37: 75-87.

Ellison, J.C. \& D.R. Stoddart. 1991. Mangrove ecosystem collapse during predicted sea level rise: Holocene analogues and implications. J. Coastal Res. 7: 151165 .

Ellison, J.C. 1996. Pollen evidence of Late Holocene mangrove development in Bermuda. Global Ecol. Biogeogr. 5: 315-326.

Ellison, J.C. 2008. Long-term restrospection on mangrove development using sediment cores and pollen analysis: A review. Aquat. Bot. 89: 93-104.

Engelhart, S.E., B.P. Horton, D.H. Roberts, C.L. Bryant \& D.R. Corbett. 2007. Mangrove pollen of Indonesia and its suitability as a sea-level indicator. Mar. Geol. 242: 65-81.

Faegri, K. \& J. Iversen. 1989. Textbook of Pollen Analysis, Wiley, Chichester, England.

Ferreira, T.O., P. Vidal-Torrado, X.L. Otero \& F. Macías. 2007. Are mangrove forest substrates sediments or soils? A case study in southeastern Brazil. Catena 70: 79-91.

Ferreyra, R. 1983. Los tipos de vegetación de la costa Peruana. Anales Jard. Bot. Madrid 40: 241-256.

Field, C.D. 1995. Impact of expected climate change on mangroves. Hydrobiologia 295: 75-81.

Gilman, E., J. Ellison \& I. Sauni Jr. 2007. Trends in surface elevation of American Samoa mangroves. Wetl. Ecol. Manage. 15: 391-404.

Gil-Torres, W. \& G. Ulloa-Delgado. 2001. Caracterización, diagnóstico de los manglares del departamento de Córdoba. Corporación Autónoma Regional de los Valles del Sinú y el San Jorge, Montería, Colombia.

Glenn, E., T.L. Tompson, R. Frye, R. Riley \& D. Baumgartner. 1995. Effects of salinity on growth and evapotranspiration of Typha domingensis. Pers. Aquat. Bot. 52: 75-91. 
González, C., L.E. Urrego, J.I. Martínez, J. Polania \& Y. Yokohama. 2010. Late Holocene mangrove dynamics in the Colombian Caribbean: a history of human and natural disturbances. The Holocene, doi: $10.1177 / 0959683610365941$.

González, V. 2003. Delta del Orinoco, p. 900-917. In M. Aguilera, A. Azocar y E. González-Jiménez (eds.). Biodiversidad en Venezuela. Fundación Polar, Ministerio de Ciencia y Tecnología y FONACIT, Caracas, Venezuela.

Goñi, M.A., M.J. Teixeira \& D.W. Perkey. 2003. Sources and distribution of organic matter in a river-dominated estuary (Winyah Bay, SC, USA). Estuar. Coast. Shelf S. 57: 1023-1048.

Grimm, E. 1987. CONISS: A Fortran 77 program for stratigraphically constrained cluster analysis by the method of incremental sum of square. Computer and Geoscience 13: 13-35.

Hernández, B. \& H. Wilches. 1990. Estudio de precipitación y caudales del río Sinú, crecida de 1988. IX Seminario Nacional de Hidráulica e Hidrología. Manizales, Colombia.

Hogarth, P.J. 1999. The Biology of Mangroves. Oxford, Nueva York, EEUU.

Hooghiemstra H., A.M. Lèzine, A.G. Suzanne, L. Dupont \& F. Marret. 2006. Late Quaternary palynology in marine sediments: A synthesis of the understanding of pollen distribution patterns in the NW African setting. Quatern. Int. 148: 29-44.

IDEAM (Instituto de Hidrología, Meteorología y Estudios Ambientales). 2001. El medio ambiente en Colombia. La atmósfera, el tiempo y el clima. Bogotá, Colombia.

IPCC (Intergovernmental panel on climate change). 2007. Climate change 2007: the physical Basis. Summary for policymakers. Contribution of Working Group 1 to the Fourth Assessment Report of the Intergovernmental Panel on Climate Change, Geneva, Switzerland. (Consultado 20 enero 2010, www.ipcc.ch/ publications and data/ar4/syr/en/spm.html).

Jurado, J. \& B. Liebmann. 2003. Relaciones entre caudales mensuales en ríos de Colombia y las temperaturas superficiales del mar en la zona tropical. Meteorol. Colomb. 7: 37-52.

Kumaran, K.P.N., M.R. Shindicar \& T.R. Mudgal. 2007. Floristic composition, palynology and sedimentary facies of Hadi mangrove swamp (Maharashtra). J. Ind. Geophys. Union 8: 55-63.

Lamb, A.L., G.P. Wilson \& M.J. Leng. 2006. A review of coastal palaeoclimate and relative sea-level reconstructions using $\mathrm{d}^{13} \mathrm{C}$ and $\mathrm{C} / \mathrm{N}$ ratios in organic material. Earth- Sci. Rev. 75: 29-57.

Mazda, Y., M. Magi, H. Nanao, M. Kogo, T. Miyagi \& N. Kanazawa. 2002. Coastal erosion due to long-term human impact on mangrove. Wetl. Ecol. Manag. 10: $1-9$.

McKee, K.L., D.R. Cahoon \& I.C. Feller. 2007. Caribbean mangroves adjust to rising sea level through biotic controls on change in soil elevation. Global Ecol. Biogeogr. 16: 545-556.

Medina, E., E. Cuevas, M. Popp \& A.E. Lugo. 1990. Soil salinity, sun exposure and growth of Acrostichum aureum, the mangrove fern. Bot. Gaz. 151: 41-49.

Mejía. T. \& P. House. 2002. Mapa de Ecosistemas Vegetales de Honduras. M.D.C. Tegucigalpa, Honduras.

Molina, A., C. Molina, L. Giraldo, C. Parra \& P. Chevillot. 1994. Dinámica marina y sus efectos sobre la geomorfología del golfo de Morrosquillo. Boletín Científico del CIOH 15: 93-113.

Neotrópicos. 1997. Identificación de actividades prioritarias para el Parque Nacional Paramillo. Informe final. Anexo 2: Aspectos Bioecológicos. Medellín, Colombia.

Nyberg, J., A. Kuijpers, B.A. Malmgren \& H. Kunzendorf. 2001. Late Holocene Changes in Precipitation and Hydrography Recorded in Marine Sediments from the Northeastern Caribbean Sea. Quaternary Res. 56: $87-102$

Nyberg, J., B.A. Malmgren, A. Kuijpers \& A. Winter. 2002. A centennial-scale variability of tropical North Atlantic surface hydrography during the late Holocene. Palaeogeogr. Palaeocl. 183: 25-41.

Olaya, H., D. Centenaro, I. Leguizamo \& F. Pineda. 1991. Los bosques de mangle en el antiguo delta del río Sinú (Córdoba-Colombia). Universidad de Córdoba, ICFES, CVS, Forestales FVE. Montería, Colombia.

Palacio, H.H. \& A.F. Restrepo. 1999. Influencia de la evolución del delta del río Sinú en los procesos morfodinámicos del litoral Caribe Antioqueño. Tesis Ingeniería Civil, Universidad Nacional de Colombia, Medellín, Colombia. 
Parkinson, R.W., R.D. de Laune \& J.R. White. 1994. Holocene Sea-Level rise and the fate of mangrove forests within the wider Caribbean region. J. Coastal Res. 10: $1077-1086$.

Parsons, J. 1952. The settlement of the Sinu valley of Colombia. Geogr. Rev. 42: 67-86.

Polissar, P.J., M.B. Abbott, A.P. Wolfe, M. Bezada, V. Rull \& R.S. Bradley. 2006. Solar modulation of Little Ice Age climate in the tropical Andes. P. Natl. Acad. Sci. USA. 103: 8937-8942.

Quiceno, M.M. 2008. Patrones de variabilidad climática y oceánica en la zona costera del río Sinú y su señal en registros naturales lacustres y coralinos. Tesis de maestría, Universidad Nacional de Colombia, Medellín, Colombia.

Robertson, K. \& J. Chaparro. 1998. Evolución histórica del río Sinú. Cuadernos de Geografía 7: 70-87.

Robertson, K. \& N. Martínez. 1999. Cambios en el nivel del mar durante el Holoceno en el litoral Caribe Colombiano. Cuadernos de Geografía 8: 168-198.

Ross, M.S., J.F. Meeder, J.P. Sah, P.I. Ruí \& G.J. Telesnicki. 2000. The Southwest Saline Everglades revisited 50 years of coastal vegetation change. J. Veg. Sci. 11: $101-112$

Ruíz-Ochoa, M., G. Bernal \& J. Polanía. 2008. Influencia del río Sinú y el mar Caribe en el sistema lagunar de Cispatá. Bol. Invest. Mar. Cost. 37: 29-49.

Rull, V. 1998. Modern and Quaternay palynological studies in the Caribbean and Atlantic coasts of northern South America: a paleoecological-oriented review. Boletín de la Sociedad Venezolana de Geólogos 23: 5-24.

Rull, V. 1999. Paleoclimatology and sea-level history in Venezuela: new data, land-sea correlations and proposals for future studies in the framework of the IGBP-PAGES project. Interciencia 24: 92-101.

Sánchez-Páez, H., G. Ulloa-Delgado, H. Tavera-Escobar \& W. Gil-Torres. 2003. Plan de manejo integral de los manglares de la zona de uso sostenible sector estuarino de la bahía de Cispatá departamento de Córdoba. Informe final. Ministerio de Ambiente, Vivienda y Desarrollo Territorial. CVS. CONIF. OIMT, Montería, Colombia.

Sánchez-Páez, H., R. Álvarez-León, F. Pinto-Nolla, A.S. Sánchez-Alférez, J.C. Pinto-Rengifo, I. García-
Hansen \& M.T. Acosta-Peñaloza. 1997. Diagnóstico y zonificación preliminar de los manglares del caribe de Colombia. Ministerio de Medio Ambiente / OIMT, Santa Fe de Bogotá, Colombia.

Sánchez-Páez, H., G. Ulloa-Delgado \& H. Tavera-Escobar. 2004. Manual sobre zonificación y planificación para el manejo sostenible los manglares, Caribe de Colombia. Proyecto PD 60/01 Rev. 1 (F) "Manejo sostenible y restauración de los manglares por comunidades locales del Caribe de Colombia”. Ministerio de Ambiente, Vivienda y Desarrollo Territorial. CONIF. OIMT, Bogotá, Colombia.

Serrano, B. 2001. Estudios de la geometría y de los cambios del delta del río Sinú durante el Holoceno. Departamento de Geociencias. Facultad de Ciencias. Universidad Nacional de Colombia, Bogotá, Colombia.

Serrano, B. 2004. The Sinú River delta on the northwestern Caribbean coast of Colombia: Bay infilling associate with delta development. J. S. Am. Earth Sci. 16: 623-631.

Snedaker, S.C. 1995. Mangroves and climate in the Florida and Caribbean region: Scenarios and hypotheses. Hydrobiology 295: 43-49.

Ter Braak, C.J.F. \& P. Smilauer. 2002. CANOCO. Reference Manual and CanoDraw for Windows User's guide: Software for Canonical Community Ordination (version 4.5). Microcomputer Power (Ithaca, Nueva York, EEUU).

Traverse, A. 1988. Paleopalynology. Unwin Hyman, Londres, Inlgaterra.

Troll, C. \& E. Schmidt. 1985. Das neue delta des Rio Sinu an der Karibischen Kuste Kolombiens. Georgraphische interpretation un kartographische Auswwertung von Luftbildern, Mit 1 Abbildung, 3 Bildern und 3.

Ulloa-Delgado, G.A., W.O. Gil-Torres, J.C. Pino-Rengifo \& H. Rodríguez-Cruz. 1998. Manual sobre técnicas de vivero y restauración de áreas de manglar del Caribe colombiano. Proyecto PD 171/91 Rev. 2 Fase II (Etapa I) Conservación y Manejo para el Uso Múltiple y el Desarrollo de los Manglares en Colombia, MMA / ACOFORE / OIMT, Santa Fe de Bogotá, Colombia.

Urrego, L.E., G. Bernal \& J. Polanía. 2009. Comparison of pollen distribution patterns in surface sediments of a Colombian Caribbean mangrove with 
geomorphology and vegetation. Rev. Palaeobot. Palyno. $156: 358-375$.

White, W., A. Warne, E. Guevara, A. Aslan, T. Tremblay \& J. Raney. 2002. Geo-environments of the northwest Orinoco Delta, Venezuela. Interciencia 27: 521-528.

Willard, D.A., L.M. Weimer \& W.L. Riegel. 2001. Pollen assemblages as paleoenvironmental proxies in the
Florida Evergaldes. Review of Palaeobotany and Palynology 113: 213-235.

Woodroffe, C.D., 1990. The impact of sea-level rise on mangrove shorelines. Prog. Phys. Geog. 14: 483-520.

Woodroffe, C.D., 1999. Response of mangrove shorelines to sea-level change. Tropics 8: 159-177. 